\title{
Evaluation of Changes In Carotid Artery Elasticity And The Associated Risk Factors In Patients With Non-Alcoholic Fatty Liver Disease By Measuring Carotid Pulse Wave Velocity Using Ultrafast Ultrasound
}

Yue Wang ( $\nabla$ wangyue_0908@163.com )

The First Affiliated Hospital of Jinzhou Medical University

Lili Ge

The First Affiliated Hospital of Jinzhou Medical University

Yuhong Li

The First Affiliated Hospital of Jinzhou Medical University https://orcid.org/0000-0003-0056-8862

\section{Research Article}

Keywords: Non-alcoholic fatty liver disease, carotid artery, elasticity, ultrafast pulse wave velocity, pulse wave velocity

Posted Date: July 7th, 2021

DOl: https://doi.org/10.21203/rs.3.rs-596009/v1

License: (9) This work is licensed under a Creative Commons Attribution 4.0 International License. Read Full License 


\section{Abstract}

Purpose: To evaluate the early changes in carotid artery elasticity in patients with non-alcoholic fatty liver disease (NAFLD) by measuring carotid ultrafast pulse wave velocity (UFPWV).

Methods: Data of 89 patients with NAFLD (observation group) and 155 healthy people (control group) were collected. The clinical and laboratory indices, carotid intima-media thickness (IMT), and PWV at the beginning of systole (PWV-BS), and the end of systole (PWV-ES) were compared and statistically analyzed.

Results: Body mass index (BMI), systolic blood pressure (SBP), diastolic blood pressure (DBP), total cholesterol (TC), triglycerides (TG), and low-density lipoprotein cholesterol (LDL-C) levels, IMT, PWV-BS, and PWV-ES in the NAFLD group were higher than those in the control group $(P<0.05)$, while the highdensity lipoprotein cholesterol (HDL-C) level was lower than that in the control group $(P<0.05)$. Moreover, IMT, PWV-BS, and PWV-ES values increased with the increasing severity of NAFLD $(P<0.05)$. Pearson correlation analysis demonstrated that PWV-BS and PWV-ES were positively correlated with age, BMI, fasting plasma glucose (FPG), SBP, DBP, TC, TG, and LDL-C $(P<0.05)$, and negatively correlated with HDL-C $(P<0.05)$. Stepwise multiple linear regression analysis indicated that the factors influencing PWVBS included BMI, FPG, TG, HDL-C, and LDL-C $(P<0.05)$, whereas the factors influencing PWV-ES included BMI, FPG, SBP, TG, HDL-C, and LDL-C $(P<0.05)$.

Conclusion: UFPWV measurement can detect the significant increase in PWV-BS and PWV-ES in patients with NAFLD. This can provide a theoretical basis for early intervention in carotid atherosclerosis.

\section{Introduction}

In recent years, the prevalence of non-alcoholic fatty liver disease (NAFLD) has increased remarkably in China due to a growing aging population, and changes in diet and lifestyle [1]. Risk factors for NAFLD are similar to those for atherosclerotic cardiovascular disease (ASCVD) [2]; therefore, individuals with NAFLD are at an increased risk for developing ASCVD, mortality, and disability [3]. Patients with NAFLD should undergo an early assessment for carotid atherosclerosis in order to prevent cardiovascular diseases. Ultrafast ultrasound imaging for carotid pulse wave velocity measurement (ultrafast pulse wave velocity, UFPWV) is a fast, accurate, and non-invasive method that provides detailed information regarding the presence of atherosclerosis in the carotid artery $[4,5]$. This study aimed to explore the changes in vascular elasticity and the factors affecting them in the patients with different severities of NAFLD at the early stage of carotid atherosclerosis by measuring UFPWV.

\section{Materials And Methods}

\subsection{Patient characteristics}


This study enrolled 89 patients, 51 males and 38 females, diagnosed with NAFLD at the physical examination center of the First Affiliated Hospital of Jinzhou Medical University from December 2020 to June 2021. These patients were included in the NAFLD group (observation group). The average age was $43.36 \pm 8.98$ years and ranged from 26-65 years. Based on the ultrasonographic findings, they were divided into mild/moderate (59 patients) and severe NAFLD groups (30 patients) [6]. This study also enrolled 155 healthy volunteers (control group) who visited the same physical examination center in the same period, and included 78 males and 77 females. The healthy participants had an average age of $43.32 \pm 8.63$ years, with a range of $25-67$ years.

Healthy individuals have a normal UFPWV as stated in the Manual of examining pulse wave velocity using Ultra-Fast Ultrasound Imaging in 2019. All patients in the NAFLD group met the diagnostic criteria of NAFLD as stated in the Guidelines for the Prevention and Treatment of non-alcoholic fatty liver disease (updated version in 2018) [1]. Diagnosis of NAFLD requires (1) liver imaging or histology consistent with the presentation of diffuse hepatocyte steatosis, (2) no history of significant alcohol consumption (alcohol intake < $210 \mathrm{~g} /$ week for a male and < $140 \mathrm{~g} /$ week for a female), and (3) absence of other diseases associated with fatty liver. These include viral hepatitis, drug-induced liver disease (due to glucocorticoid, estrogen, methotrexate use, etc.), autoimmune liver disease, hepatolenticular degeneration, total parenteral nutrition, inflammatory bowel disease, celiac disease, hypothyroidism, Cushing's syndrome, abetalipoproteinemia, lipoatrophic diabetes, and Mauriac syndrome. Abdominal ultrasound was used for liver imaging for the diagnosis of NAFLD, and all the patients met the diagnostic criteria stated in the Guidelines for the diagnosis and treatment of nonalcoholic fatty liver disease and alcoholic liver disease (2006 version) [6]. According to the criteria, (1) the liver size is normal, or there is only mild to moderate liver enlargement. The patient has a blunted liver edge. Moreover, a small up and down movement in the liver is observed during breathing. (2) The echo in the liver's near-field, which is diffuse and dense, is stronger ("bright liver echo") than that of the kidney and spleen. Gradual attenuation of the far-field echo is present, which seems to have a "haze-like" visual effect. (3) The number of blood vessels in the liver is obviously reduced and their texture is unclear. The hepatic and/or portal vein branches are hypoechoic, and punctate echoes are observed in the portal vein. (4) Color flow Doppler imaging displays decreased color flow signal in the liver or arbitrary blood flow, but the blood vessels in the liver are normal. (5) The echo of the right lobe capsule and diaphragm is unclear and incomplete, and the adjacent boundary with the gallbladder and right kidney is blurred. Diagnosis of mild fatty liver requires the presence of point (1) and any one of the point from (2) to (4). Diagnosis of moderate fatty liver requires the presence of point (1) and any two of the points from (2) to (4). Diagnosis of severe fatty liver requires the presence of points (1), (5), and any two of the points from (2) to (4).

The exclusion criteria for the control group were (1) carotid intima-media thickening (IMT) $\geq 0.1 \mathrm{~cm}$ or plaque formation (IMT $\geq 0.15 \mathrm{~cm}$ ) [7]; (2) history of malignancy, severe hepatorenal insufficiency, heart failure, obesity (body mass index $[\mathrm{BMI}] \geq 30 \mathrm{~kg} / \mathrm{m}^{2}$ ), diabetes, and peripheral vascular diseases; (3) taking drugs that could affect the vasomotor function for a week; and (4) the failure of UFPWV 
measurement due to examination difficulties (large breathing amplitude, being unable to hold breath, tachycardia), thick neck, deeply positioned or short carotid artery, and abnormal arterial tortuosity.

The study was approved by the local Ethical Committee, and written informed consent was obtained from each participant.

\subsection{Instruments and methods}

The Supersonic Imagine Aixplorer (SuperSonic Imagine, Aix-en-Provence, France) ultrasound diagnostic device with built-in ability to measure UFPWV was used in this study. The device setting for carotid assessment was used and an SL10-2 linear probe having a frequency of 6-9 $\mathrm{MHz}$ was employed. The participants were asked to lie on their back, with their heads rotated $45^{\circ}$ toward the non-examination side to fully expose the neck. The probe was positioned to perform the longitudinal scan of the carotid artery. The largest section of the long axis of the common carotid artery (about 1-2 cm away from the carotid sinus) was assessed [8]. IMT was measured when the interfaces between the blood and intima and the media and adventitia were visualized. Then the participants were asked to hold their breath. PWV was measured when the image became stable. PWV at the beginning of systole (PWV-BS), at the end of systole (PWV-ES), and their standard deviation (SD) were automatically measured (Fig. 1-3). UFPWV measurement with SD $\leq 20 \%$ was considered accurate. Bilateral carotid arteries of each participant were measured three times and the mean value was taken. All the measurements were taken by experienced ultrasonologists.

\subsection{Reproducibility Testing}

Forty individuals were randomly selected from all the participants. Two observers repeated the measurements of PWV-BS and PWV-ES to evaluate the inter- and intra-observer reliability and reproducibility. Intraclass correlation coefficient values (ICC) $>0.75$ indicated good reliability.

\subsection{Statistical analysis}

SPSS 23.0 (IBM Corp., Armonk, NY, USA) was used for statistical analysis. Normally distributed continuous data were expressed as mean \pm SD. Independent sample t-test was used to compare the means of the NAFLD group with that of the control group. Categorical data were represented as percentages (\%) and were compared by using the $\chi^{2}$ test. Pearson correlation analysis was used as well. The associated factors were analyzed by stepwise multiple linear regression analysis. Receiver operating characteristic curve (ROC curve) was drawn for the BS and ES values. The sensitivity and specificity of the different cut-off values were calculated using the area under the curve (AUC), and the critical value was determined to be the reference value. A $P$-value $\leq 0.05$ was considered statistically significant.

\section{Results}

\subsection{Reproducibility testing}


The results of reproducibility testing demonstrated that the inter- and intra-observer ICC values of PWV-BS and PWV-ES were more than 0.75 , indicating good reliability.

\subsection{Comparison of clinical and laboratory indices between the NAFLD and the control group}

BMI, systolic blood pressure (SBP), diastolic blood pressure (DBP), total cholesterol (TC), triglycerides (TG) and low-density lipoprotein cholesterol (LDL-C) levels in the NAFLD group were higher than those in the control group $(P<0.05)$, while the level of HDL-C in the NAFLD group was lower than the control group $(P<0.05)$. There were no significant differences in age, gender and fasting plasma glucose (FPG) between the two groups $(P>0.05)$ (Table 1$)$.

Table 1

Comparison of clinical and laboratory indices between the NAFLD and the control group $(x \pm S D)$

\begin{tabular}{|c|c|c|c|c|}
\hline Group & $\operatorname{NAFLD}(n=89)$ & Control $(n=155)$ & $t / \chi^{2}$ value & P-value \\
\hline Age (years) & $43.36 \pm 8.98$ & $43.32 \pm 8.63$ & 0.032 & 0.975 \\
\hline Male/Female & $51 / 38$ & $78 / 77$ & 1.106 & 0.351 \\
\hline $\mathrm{BMI}\left(\mathrm{kg} / \mathrm{m}^{2}\right)$ & $24.87 \pm 2.56$ & $21.56 \pm 2.18$ & 10.249 & $<0.01$ \\
\hline $\mathrm{FPG}(\mathrm{mmol} / \mathrm{L})$ & $4.95 \pm 0.56$ & $4.87 \pm 0.62$ & 1.018 & 0.310 \\
\hline $\mathrm{SBP}(\mathrm{mmHg})$ & $123.82 \pm 8.50$ & $117.11 \pm 8.43$ & 5.967 & $<0.01$ \\
\hline $\mathrm{DBP}(\mathrm{mmHg})$ & $76.33 \pm 6.63$ & $74.50 \pm 5.34$ & 2.220 & 0.028 \\
\hline $\mathrm{TC}(\mathrm{mmol} / \mathrm{L})$ & $4.81 \pm 0.88$ & $4.29 \pm 0.51$ & 5.127 & $<0.01$ \\
\hline $\mathrm{TG}(\mathrm{mmol} / \mathrm{L})$ & $1.83 \pm 0.86$ & $1.06 \pm 0.29$ & 8.192 & $<0.01$ \\
\hline $\mathrm{HDL}-\mathrm{C}(\mathrm{mmol} / \mathrm{L})$ & $1.28 \pm 0.38$ & $1.85 \pm 0.44$ & -10.529 & $<0.01$ \\
\hline LDL-C (mmol/L) & $3.10 \pm 1.08$ & $2.31 \pm 0.53$ & 6.437 & $<0.01$ \\
\hline IMT (mm) & $0.61 \pm 0.13$ & $0.53 \pm 0.10$ & 5.259 & $<0.01$ \\
\hline PWV-BS (m/s) & $7.27 \pm 1.33$ & $5.79 \pm 1.03$ & 9.106 & $<0.01$ \\
\hline PWV-ES (m/s) & $9.49 \pm 1.11$ & $7.12 \pm 0.99$ & 17.210 & $<0.01$ \\
\hline
\end{tabular}

\subsection{Comparison of IMT, PWV-BS, and PWV-ES}

No significant differences were reported in IMT, PWV-BS, and PWV-ES between the left and right common carotid arteries in the mild/moderate NAFLD, severe NAFLD, and the control group $(P>0.05)$. Therefore, 
the mean values obtained from the bilateral carotid arteries were used. There were significant differences in IMT, PWV-BS, and PWV-ES among the mild/moderate, and severe NAFLD, and the control groups $(P<$ 0.05). IMT, PWV-BS, and PWV-ES in the NAFLD group were higher than those in the control group $(P<$ 0.05). Comparison between the different NAFLD groups showed that the severe NAFLD group had higher values of IMT, PWV-BS, and PWV-ES than those in the mild/moderate group $(P<0.05)($ Table 2$)$.

Table 2

Comparison of PWV-BS and PWV-ES between the NAFLD and the control group $(x \pm S D)$

\begin{tabular}{|c|c|c|c|}
\hline Group & IMT (mm) & PWV-BS (m/s) & PWV-ES (m/s) \\
\hline Control $(n=155)$ & $0.53 \pm 0.10$ & $5.79 \pm 1.03$ & $7.12 \pm 0.99$ \\
\hline Mild/moderate $(n=59)$ & $0.58 \pm 0.12^{\star \#}$ & $6.86 \pm 1.30^{\star \#}$ & $9.16 \pm 1.11^{\star \#}$ \\
\hline Severe $(n=30)$ & $0.67 \pm 0.12^{\star \#}$ & $8.08 \pm 1.00^{\star \#}$ & $10.15 \pm 0.79^{\star \#}$ \\
\hline F-value & 23.24 & 64.57 & 169.30 \\
\hline P-value & $<0.01$ & $<0.01$ & $<0.01$ \\
\hline \multicolumn{4}{|c|}{$\begin{array}{l}{ }^{\star} P<0.01 \text { when comparing with the control group }<;{ }^{\#} P<0.01 \text { when comparing with the mild/moderate } \\
\text { group }<\end{array}$} \\
\hline
\end{tabular}

\subsection{Pearson correlation analysis}

PWV-BS and PWV-ES were positively correlated with age, BMI, FPG, SBP, DBP, TC, TG, and LDL-C ( $P<$ $0.05)$, and negatively correlated with HDL-C $(P<0.05)$ (Table 3$)$. 
Table 3

Factors affecting PWV-BS and PWV-ES in NAFLD patients $(\mathrm{n}=89)$

\begin{tabular}{|c|c|c|c|c|}
\hline \multirow[t]{2}{*}{ Risk factors } & \multicolumn{2}{|c|}{ PWV-BS } & \multicolumn{2}{|c|}{ PWV-ES } \\
\hline & $r$ & $P$-value & $\mathbf{r}$ & $P$-value \\
\hline Age & 0.215 & $<0.01$ & 0.195 & $<0.01$ \\
\hline BMI & 0.557 & $<0.01$ & 0.626 & $<0.01$ \\
\hline FPG & 0.324 & $<0.01$ & 0.241 & $<0.01$ \\
\hline SBP & 0.498 & $<0.01$ & 0.539 & $<0.01$ \\
\hline DBP & 0.363 & $<0.01$ & 0.342 & $<0.01$ \\
\hline TC & 0.371 & $<0.01$ & 0.377 & $<0.01$ \\
\hline TG & 0.475 & $<0.01$ & 0.573 & $<0.01$ \\
\hline HDL-C & -0.501 & $<0.01$ & -0.593 & $<0.01$ \\
\hline LDL-C & 0.563 & $<0.01$ & 0.559 & $<0.01$ \\
\hline \multicolumn{5}{|c|}{$\begin{array}{l}\text { BMI, body mass index; NAFLD, non-alcoholic fatty liver disease; PWV-BS, pulse wave velocity- } \\
\text { beginning of systole; PWV-ES, pulse wave velocity-end of systole; TC, total cholesterol; TG, } \\
\text { triglycerides; LDL-C, low-density lipoprotein cholesterol; IMT, intima-media thickness; HDL-C, high- } \\
\text { density lipoprotein cholesterol; FPG, fasting plasma glucose; SBP, systolic blood pressure; DBP, } \\
\text { diastolic blood pressure }\end{array}$} \\
\hline
\end{tabular}

\subsection{Analysis of factors affecting PWV-BS and PWV-ES}

PWV-BS and PWV-ES were used as dependent variables, while age, BMI, FPG, SBP, DBP, TC, TG, HDL-C, and $L D L-C$ were used as independent variables to conduct stepwise multiple linear regression analysis. The results showed that the factors affecting PWV-BS included BMI, FPG, TG, HDL-C, and LDL-C ( $P<$ 0.05), whereas the factors affecting PWV-ES included BMI, FPG, SBP, TG, HDL-C, and LDL-C $(P<0.05)$ (Table 4). 
Table 4

Stepwise multiple linear regression analysis $(n=89)$

\begin{tabular}{|c|c|c|c|c|}
\hline \multirow[t]{2}{*}{ Independent variables } & \multicolumn{2}{|c|}{ PWV-BS } & \multicolumn{2}{|c|}{ PWV-ES } \\
\hline & $\beta$ & $t$ & $\beta$ & $\mathrm{t}$ \\
\hline BMI & 0.132 & $5.710^{\star}$ & 0.173 & $7.413^{*}$ \\
\hline FPG & 0.527 & $5.577^{*}$ & 0.373 & $3.914^{*}$ \\
\hline SBP & 0.010 & 0.949 & 0.021 & $2.043^{\#}$ \\
\hline TG & 0.278 & $2.765^{\star}$ & 0.503 & $4.967^{\star}$ \\
\hline HDL-C & -0.438 & $-3.238^{*}$ & -0.719 & $-5.276^{*}$ \\
\hline LDL-C & 0.389 & $4.733^{*}$ & 0.352 & $4.256^{*}$ \\
\hline \multicolumn{5}{|l|}{${ }^{*} P<0.01 ;{ }^{\#} P<0.05$} \\
\hline
\end{tabular}

\subsection{ROC curves}

The areas under the ROC curves for PWV-BS and PWV-ES were 0.81 and 0.94 , respectively (all $P<0.05$ ). The optimal cut-off value for PWV-BS was $6.53 \mathrm{~m} / \mathrm{s}$. When this value was used as reference, PWV-BS was able to detect carotid artery atherosclerosis with a sensitivity of $76.4 \%$ and a specificity of $77.4 \%$. The optimal cut-off value, at which PWV-ES had a sensitivity of $83.1 \%$ and a specificity of $91.6 \%$ for detecting carotid artery atherosclerosis, was $8.47 \mathrm{~m} / \mathrm{s}$ (Fig. 4).

\section{Discussion}

NAFLD is a clinicopathological syndrome characterized by hepatocyte steatosis and abnormal accumulation of fat (mainly TG) in the absence of secondary causes, such as excessive consumption of alcohol and other factors that cause liver injury $[1,5]$. Clinically, carotid atherosclerosis is often accidentally discovered in patients with NAFLD on physical examination. Atherosclerosis is a complex disease process, caused by a combination of structural and functional changes occurring in the arterial walls [4]. Structural changes include build-up of lipids, intimal thickening and plaque formation in the arterial walls, which can be observed by conventional ultrasonographic measurement of IMT. Functional changes occur before the structural changes, and include arterial stiffness and medial hypertrophy due to the gradual increase in blood pressure. It is impossible to differentiate between intimal thickening caused by early plaque formation or functional changes occurring in the arterial wall on the basis of IMT measurement. Therefore, PWV needs to be measured to assess early vascular changes [9]. 
UFPWV plays an important role in the evaluation of early arteriosclerosis. With a frame rate as high as 10,000 frames/s, ultrafast ultrasound imaging technology can quickly (within 2 seconds) record the pulse wave propagation in the carotid artery wall and measure PWV-BS and PWV-ES [10]. Assuming that the carotid artery acts like a cylindrical elastic tube filled with incompressible fluid, the relationship between Young's modulus and PWV is calculated by the Moens-Korteweg equation:

$P W V=\sqrt{\frac{E h}{2 \rho R\left(+v^{2}\right)}}$, where $E$ is the Young's modulus of vascular wall, $v$ is Poisson's ratio, $h$ is the vessel wall thickness, $\rho$ is the blood density, and $R$ is the vessel radius [11]. According to this formula, PWV is inversely proportional to vascular elasticity, that is, patients with fast PWV have carotid arteries with poor elasticity due to the hardening of the arterial walls [10]. Therefore, PWV can be a useful indicator of arteriosclerosis in the early stage, and can help in evaluating the risk for ASCVD $[10,12,13]$.

Increased levels of TG and LDL-C affect liver and blood vessels similarly, and there is a potential linkage between NAFLD and ASCVD [14]. In this study, patients with NAFLD were divided into mild/moderate and severe NAFLD groups on the basis of abdominal ultrasound examination findings. Moreover, a comparison was made between the NAFLD and the control group in terms of the IMT, PWV-BS, and PWVES values. Results showed that IMT values increased with the increase in the severity of NAFLD, which is consistent with the findings of a recent 18-month long European study [15]. This study indicated that the improvement in NAFLD severity was independently associated with the decrease in carotid IMT progression. It also showed that the values of PWV-BS and PWV-ES in patients with NAFLD were significantly higher than those in the healthy cohort. In addition, PWV-BS and PWV-ES values in patients with severe NAFLD were higher than those in patients with mild/moderate NAFLD, which proves that the elasticity of the arterial walls in the patients with NAFLD is significantly lower than that of the arteries of healthy people before the morphological changes, characteristic of atherosclerosis, appear in the carotid artery. Moreover, atherosclerosis worsens with the worsening of NAFLD, which is consistent with the findings of Alkhouri et al. [16]. They showed that the increased severity of NAFLD was associated with abnormal lipid metabolism, which resulted in a hypercoagulable state, thus leading to a higher risk of ASCVD. A previous clinical trial [17] provided evidence that the treatment of NAFLD is beneficial in preventing ASCVD. Moreover, Luan et al. stated that $20-80 \%$ of NAFLD cases have dyslipidemia because NAFLD stimulates free fatty acid oxidation and secretion of very-low-density lipoprotein by the liver [18]. Our study also shows that the TC, TG, and LDL-C levels in patients with NAFLD were higher than those in healthy people, while HDL-C level was lower. Increased severity of hepatic steatosis is probably associated with a higher risk of atherosclerosis because of the increase in the plasma levels of TC, TG, and LDL-C and reduction in the HDL-C level, which is consistent with the findings of the previous studies. It is assumed that hyperlipidemia, chronic inflammation, and oxidative stress induced by NAFLD (i.e. accumulation of TG in hepatocytes) activate endothelial and inflammatory cells, which produce local chemokines [19]. These chemokines accelerate vascular endothelial damage and proliferation of the arterial wall smooth muscle cells, resulting in decreased arterial wall compliance. IMT gradually increases with the increased severity of NAFLD, due to the atherosclerotic plaque formation, vascular stenosis, 
rupture of unstable plaques, and hemodynamic alterations in the carotid blood flow. As a result, turbulence and shear stress cause intimal damage, which together with lipid deposition further accelerate carotid atherosclerosis. Due to decreased perfusion of cerebral tissues, patients may experience transient ischemic attacks or stroke.

This study also evaluates the factors affecting PWV-BS and PWV-ES in the NAFLD group. Results showed that PWV-BS and PWV-ES were positively correlated with age, BMI, FPG, SBP, DBP, TC, TG, and LDL-C $(P<0.05)$, and negatively correlated with HDL-C. Older age, hypertension, impaired glucose tolerance, dyslipidemia, and obesity are the risk factors for hemodynamic abnormalities, metabolic syndrome, as well as atherosclerosis. These factors lead to vascular endothelial dysfunction through different pathways. Previous studies $[13,20,21]$ also show that the abovementioned factors can affect PWV-BS and PWV-ES values to varying degrees. Due to the relatively high sensitivity and specificity of UFPWV, and it quick and non-invasive nature, it may be used in clinical settings when assessing NAFLD patients.

A major limitation of this study is the use of imaging instead of liver biopsy for establishing the diagnosis of NAFLD. Imaging is not the gold standard for NAFLD diagnosis. Another limitation is the small sample size and short study time. A large multicenter study is required in the future to verify this study's results, and ascertain whether preventing the development of NAFLD or reducing its severity has a positive effect on the vascular wall. Furthermore, future researchers could conduct a comparative study between ultrafast ultrasound and other imaging modalities for measuring PWV in order to determine the most accurate method. Finally, IMT was measured manually rather than automatically, which may lead to errors in measurement. However, inter- and intra-observer reproducibility testing showed that measurements obtained via ultrafast ultrasound were reliable.

In conclusion, increased NAFLD severity may be associated with a higher risk of cardiovascular complications. UFPWV can be used as a parameter for determining a better treatment plan to slow down the progression of NAFLD and ASCVD. It is suggested that high-risk groups should consume a balanced diet, reduce sedentary habits, and exercise regularly in order to improve their quality of life. In addition, regular monitoring of blood lipid levels and liver fat content in patients with NAFLD can help detect risk factors early and delay the occurrence and development of complications.

\section{Declarations}

Funding: This study is supported by Liaoning Revitalization Talents Program (XLYC1802049).

Competing interests: The authors declare that they have no conflict of interest.

Code availability: Not applicable

Authors' contributions: YW, LLG, YHL: Conception and design, collection and analysis of data, drafting and final approval of manuscript. 
Ethics approval: The study was approved by the local Ethical Committee, and written informed consent was obtained from each participant. (Ethics approval number:202061)

Consent to participate: Not applicable

Consent for publication: Not applicable

Acknowledgement: We would like to thank Editage for English language editing of the manuscript.

\section{References}

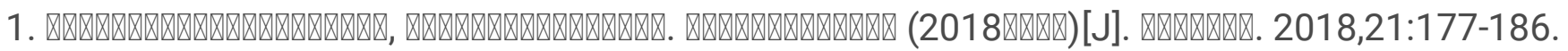

2. Luan Rodrigues Abdallah et al. Non-alcoholic Fatty Liver Disease and Its Links with Inflammation and Atherosclerosis[J]. Current Atherosclerosis Reports, 2020, 22(2) : 2095-2128.

3. Lee Hyeok-Hee, et al. Non-alcoholic steatohepatitis and progression of carotid atherosclerosis in patients with type 2 diabetes: a Korean cohortstudy.[J].Cardiovascular diabetology, 2020, 19(1) : 81.

4. Li Xiaopeng, Jiang Jue, Zhang Hong, et al. Measurement of carotid pulse wave velocity using ultrafast ultrasound imaging in hypertensive patients.2017, 44(2):183-190.

5. Fu Xiao Pan MD et al. Carotid Artery Stiffness Assessment by Ultrafast Ultrasound Imaging: Feasibility and Potential Influencing Factors[J]. Journal of Ultrasound in Medicine, 2018, 37(12) : 2759-2767.

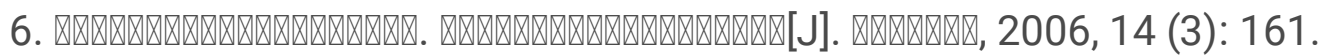

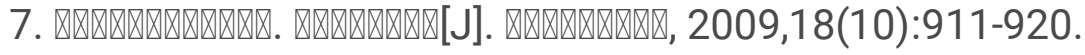

8. Lee Whal. General principles of carotid Doppler ultrasonography.[J]. Ultrasonography (Seoul, Korea),2014,33(1).

9. Reinehr T, Wunsch R, de Sousa G, et al. Relationship between metabolic syndrome definitions for children and adolescents and intima-media thickness. Atherosclerosis. 2008;199(1):193-200.

10. Zheng-Qiu Zhu, Ling-Shan Chen, Xue-Zhong Jiang, et al. Absent atherosclerotic risk factors are associated with carotid stiffening quantified with ultrafast ultrasound imaging. 2020, :1-12.

11. Nichols W, O'Rourke M. McDonald's Blood Flow in Arteries: Theoretical, Experimental and Clinical Principles. 5th edn, Hodder Arnold Publication: London, 2005, pp 57-58.16.

12. Ben-Shlomo Y, Spears $M$, Boustred $C$, et al. Aortic pulse wave velocity improves cardiovascular event prediction: an individual participant meta-analysis of prospective observational data from 17,635 subjects[J]. J Am Coll Cardiol. 2014.63:636-646.

13. W-C Yu et al. Brachial-ankle vs carotid-femoral pulse wave velocity as a determinant of cardiovascular structure and function[J]. Journal of Human Hypertension, 2008, 22(1): 24-31.

14. Loria P, Lonardo A, Targher G. Is liver fat detrimental to vessels?: intersections in the pathogenesis of NAFLD and atherosclerosis. Clin Sci (Lond) 2008;115(1):1e12. 
15. Bhatia L, Scorletti E, Curzen N, Clough GF, Calder PC, Byrne CD. Improvement in non-alcoholic fatty liver disease severity is associated with a reduction in carotid intima-media thickness progression. Atherosclerosis. 2016 Mar; 246:13-20.

16. N. Alkhouri, T. A. R. T amimi, L. Y erian, R. Lopez, N. N. Zein, and A. E. Feldstein, "The inflamed liver and atherosclerosis: a Link between histologic severity of nonalcoholic fatty liver disease and increased cardiovascular risk," Digestive Diseases and Sciences, vol. 55, no. 9, pp. 2644-2650, 2010.

17. Raul D. Santos and Luca Valenti and Stefano Romeo. Does nonalcoholic fatty liver disease cause cardiovascular disease? Current knowledge and gaps[J]. Atherosclerosis, 2019, $282:$ 110-120.

18. Luan Rodrigues Abdallah et al. Non-alcoholic Fatty Liver Disease and Its Links with Inflammation and Atherosclerosis[J]. Current Atherosclerosis Reports, 2020, 22(2) : 2095-2128.

19. GILL C, VATVHEVA K P, PAN J J, et al. Frequency of nonalcoholic fatty liver disease and subclinical atherosclerosis among young mexicanamericans[J]. Am J Cardiol, 2017, 119(11): 1717-1722.

20. Yang Weiwei et al. Establishing normal reference value of carotid ultrafast pulse wave velocity and evaluating changes on coronary slow flow. [J]. The international journal of cardiovascular imaging, 2020,36(10):1931-1939.

21. TSUCHIKURA S, SHOJI T, KIMOTO E, et al. Brachial - ankle pulsewave velocity as an index of central arterial stiffness[J]. J AtherosclerThromb, 2010, 17 (6): 658 - 665. DOI: 10.5551/jat.3616.

\section{Figures}




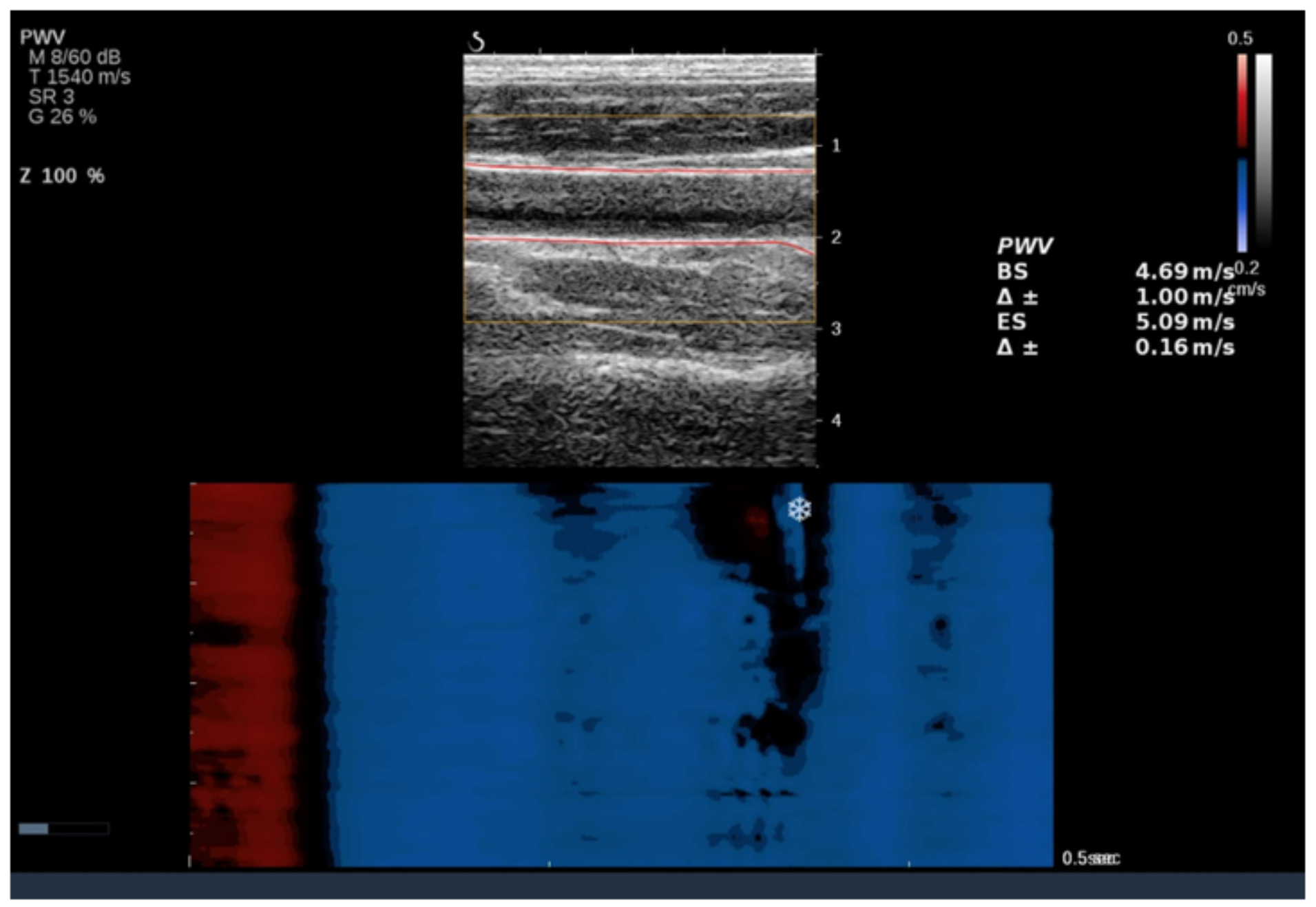

\section{Figure 1}

Measurement of pulse wave velocity (PWV) of a healthy volunteer (male, age 32, BMI 21.50 kgm-2) using ultrafast ultrasound The values of PWV-BS and standard deviation are 4.69 and 1.00 ms-1, respectively. The values of PWV-ES and standard deviation are 5.09 and $0.16 \mathrm{~ms}-1$, respectively. BMI, body mass index; PWV-BS, pulse wave velocity-beginning of systole; PWV-ES, pulse wave velocity-end of systole; $\Delta \pm$, standard deviation 


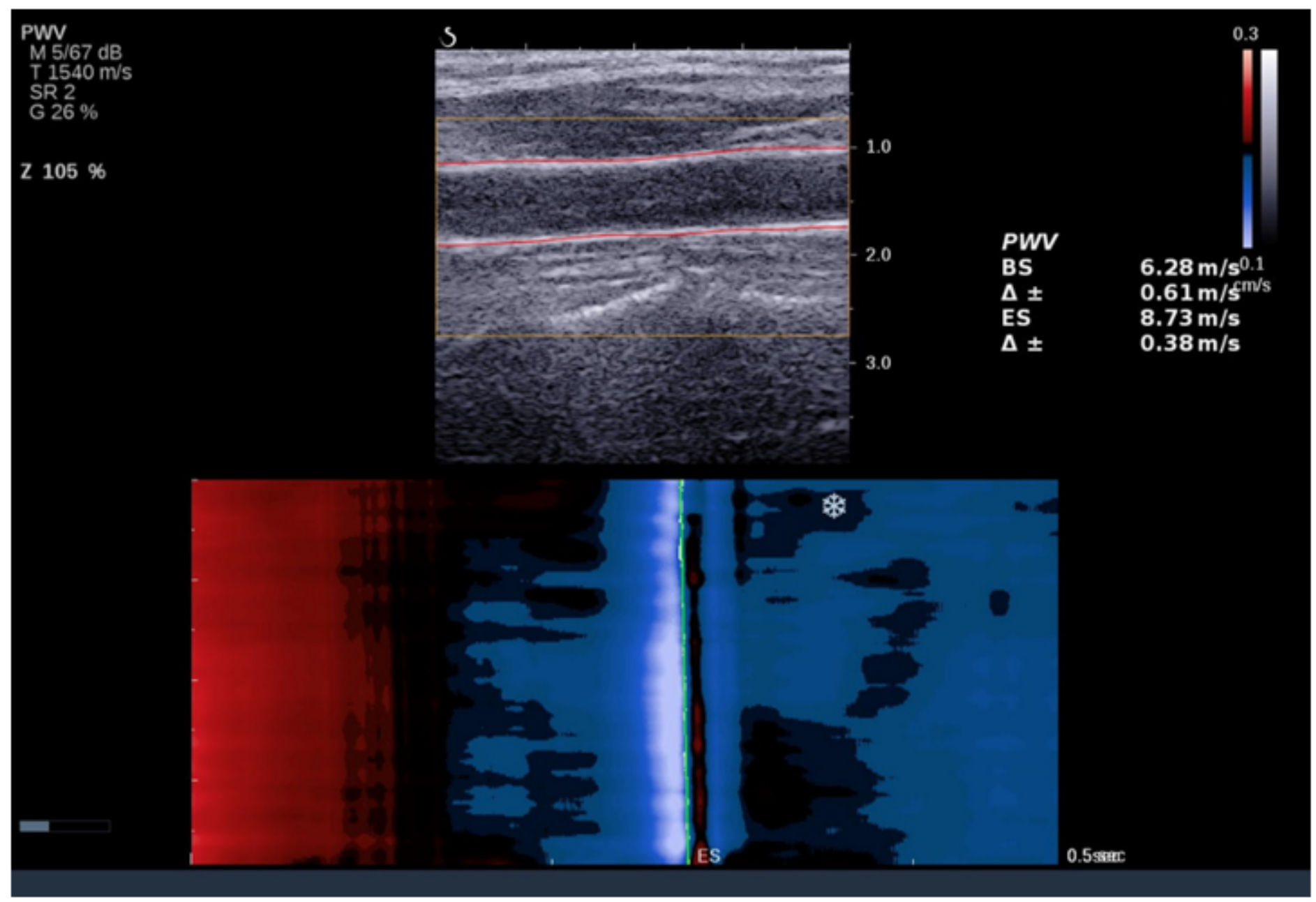

\section{Figure 2}

Measurement of pulse wave velocity (PWV) of a patient with mild-moderate NAFLD (female, age 45, BMI $24.63 \mathrm{kgm}-2$ ) using ultrafast ultrasound The values of PWV-BS and standard deviation are 6.28 and 0.61 $\mathrm{ms}-1$, respectively. The values of PWV-ES and standard deviation are 8.73 and $0.38 \mathrm{~ms}-1$, respectively. BMI, body mass index; NAFLD, non-alcoholic fatty liver disease; PWV-BS, pulse wave velocity-beginning of systole; PWV-ES, pulse wave velocity-end of systole; $\Delta \pm$, standard deviation 


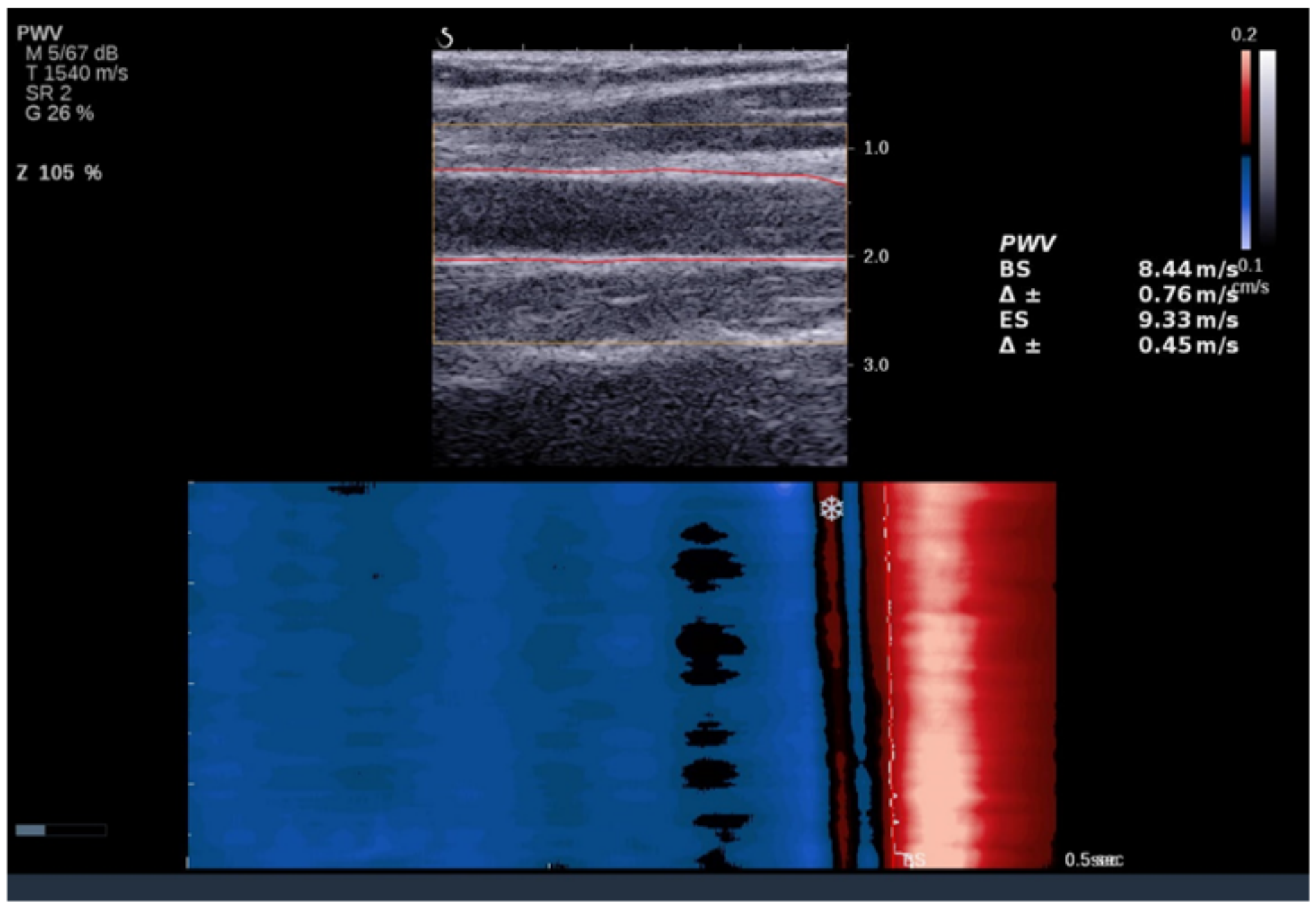

\section{Figure 3}

Measurement of pulse wave velocity (PWV) of a patient with severe NAFLD (male, age 42, BMI 27.51 kgm-2) using ultrafast ultrasound The values of PWV-BS and standard deviation are 8.44 and 0.76 ms-1, respectively. The values of PWV-ES and standard deviation are 9.33 and $0.45 \mathrm{~ms}-1$, respectively. BMI, body mass index; NAFLD, non-alcoholic fatty liver disease; PWV-BS, pulse wave velocity-beginning of systole; PWV-ES, pulse wave velocity-end of systole; $\Delta \pm$, standard deviation 

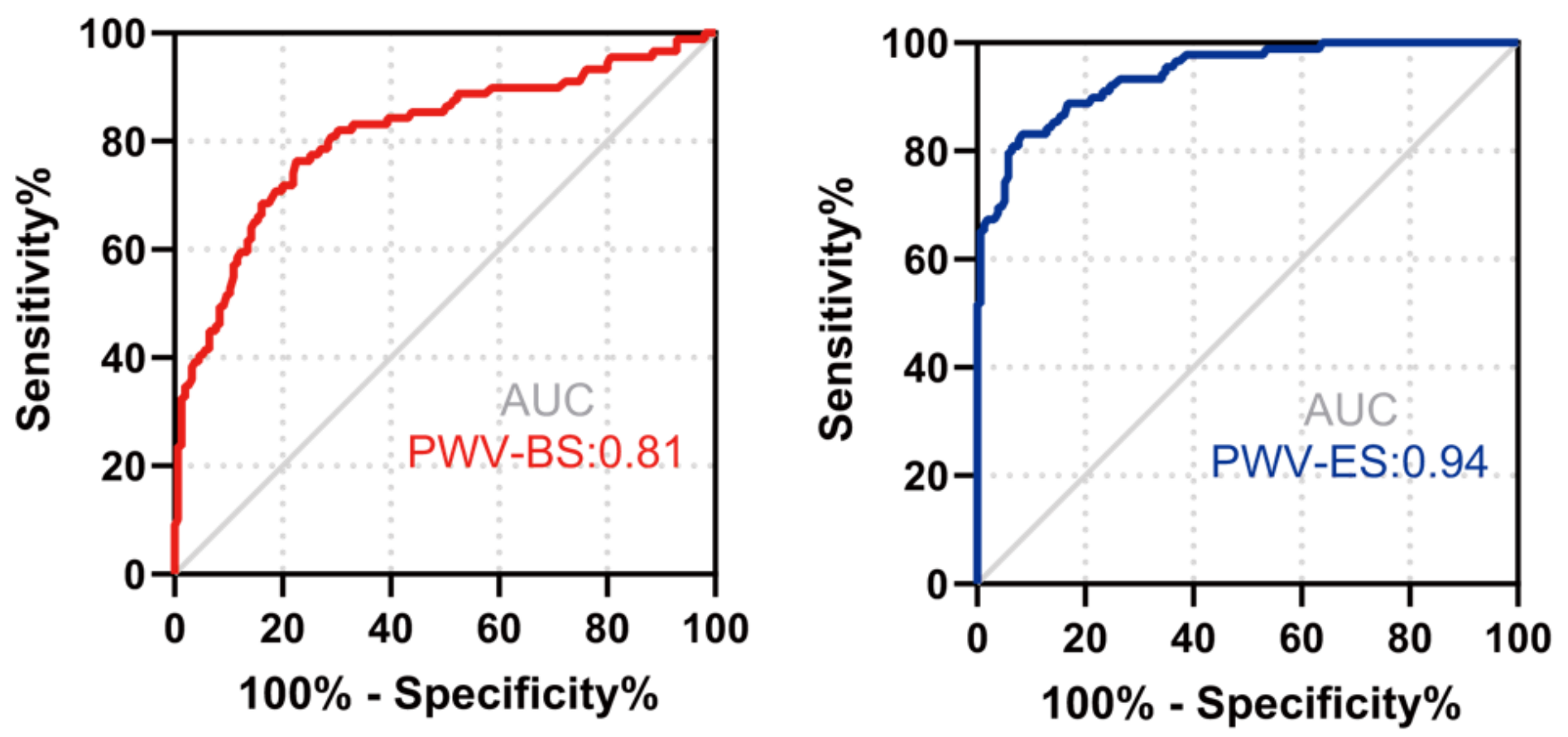

Figure 4

ROC curves evaluating the diagnostic accuracy of PWV-BS and PWV-ES in detecting the change in carotid artery elasticity among the patients with NAFLD ROC curve, receiver operating characteristic curve; PWVBS, pulse wave velocity-beginning of systole; PWV-ES, pulse wave velocity-end of systole; NAFLD, nonalcoholic fatty liver disease 